\title{
Estudo experimental da fluidodinâmica do bagaço de laranja em leito fluidizado
}

\author{
Experimental study on the fluid dynamics of orange bagasse in fluidized bed \\ T. L. C. Gomes; J. S. Lobo; T. E. A. Carvalho; M. M. Prado* \\ Departamento de Engenharia Química/Laboratório de Fenômenos de Transporte em Sistemas Particulados/CCET, \\ Universidade Federal de Sergipe, 49100-000, São Cristóvão-SE, Brasil
}

*manoelprado@ufs.br

(Recebido em 15 de março de 2016; aceito em 22 de abril de 2016)

\begin{abstract}
A laranja é uma das frutas mais utilizadas mundialmente para a produção de suco, sendo $50 \%$ dela descartada na forma de bagaço. Para o aproveitamento desse resíduo como biomassa para a geração de energia é necessária a redução do seu elevado teor de umidade. Visando avaliar o potencial uso da técnica de leito fluidizado para a secagem do bagaço de laranja, é de fundamental importância compreender o comportamento fluidodinâmico do material dentro do leito, no intuito de conhecer a capacidade de processamento e as condições de operação ótimas do equipamento. Logo, neste trabalho foram determinadas as características fluidodinâmicas do bagaço de laranja em leito fluidizado convencional, avaliando-se como os parâmetros de fluidização foram influenciados pelo tamanho de partícula e carga do material no leito. Os ensaios fluidodinâmicos foram realizados com cargas do leito de 400,700 e $1000 \mathrm{~g}$, e diâmetros médios de partícula de 1,55; 1,85 e 2,58 mm. Ao aumentar o diâmetro da partícula de 1,55 para $2,58 \mathrm{~mm}$, a velocidade de mínima fluidização $\left(\mathrm{v}_{\mathrm{mf}}\right)$ aumentou de 1,36 para $1,83 \mathrm{~m} / \mathrm{s}$. Apesar de requererem uma maior velocidade do ar para começar a fluidizar, partículas com diâmetro de 2,58 mm apresentaram o maior índice de fluidização e o menor número de Froude, sendo as mais adequadas para a operação em leito fluidizado. Ao aumentar a carga de partículas de $2,58 \mathrm{~mm}$ alimentadas no leito de 0,4 para $1 \mathrm{~kg}$, houve um aumento de $126 \%$ na queda de pressão de mínima fluidização. Entretanto, nenhuma variação significativa foi verificada em $\mathrm{v}_{\mathrm{mf}}$.

Palavras-chave: bagaço de laranja, leito fluidizado, velocidade de mínina fluidização.
\end{abstract}

Orange is widely used worldwide for juice production, and in this process almost half the fruit is discarded as residue. The use of orange bagasse as biomass for energy production requires the reduction of its high moisture content. Aiming to evaluate the potential use of the fluidized bed technology for drying orange bagasse, it is essential to understand the hydrodynamics behavior of the material inside the bed, in order to know both the processing capability and optimum operating conditions of the equipment. Thereby, in this work the hydrodynamics characteristics of the orange bagasse were determined. How the fluidization parameters were affected by particle size and bed load were also evaluated. Experiments were conducted with bed loads of 400, 700 and $1000 \mathrm{~g}$, and average particle diameter of 1,55; 1,85 e 2,58 mm. Increasing the particle diameter from 1,55 to $2,58 \mathrm{~mm}$ led to an increase in the minimum fluidization velocity ( $\mathrm{v}_{\mathrm{mf}}$ ) from 1.36 to $1.83 \mathrm{~m} / \mathrm{s}$. Although particles having diameter of $2.58 \mathrm{~mm}$ require a higher air velocity to start fluidization, they presented the highest value of fluidization index and the lowest value of Froude number, thus being the most adequate for the fluidized bed operation. The pressure drop at the minimum fluidization increased $126 \%$ with the increase in the mass of $2.58 \mathrm{~mm}$ particles fed into the bed from 0.4 to $1 \mathrm{~kg}$. However, there is no significant change in the minimum fluidization velocity for the different bed loads.

Keywords: orange bagasse, fluidized bed, minimum fluidization velocity.

\section{INTRODUÇÃO}

Líder no ranking mundial, o Brasil é responsável por, aproximadamente, $30 \%$ da produção de laranja in natura e por $60 \%$ da produção de suco de laranja de todo o planeta. O sistema agroindustrial da laranja é um caso de sucesso no país, onde a fruta atende cerca de $50 \%$ da demanda e $75 \%$ das transações internacionais, trazendo anualmente mais de US\$1 bilhão de dólares em divisas para o Brasil, no centro de uma cadeia produtiva que gera um PIB equivalente a US $\$ 5$ bilhões de dólares [1]. 
Apesar do seu potencial econômico na agroindústria brasileira, os resíduos gerados após o processamento da laranja têm causado grande preocupação para as indústrias e órgãos governamentais, já que estes representam perdas de matéria-prima e energia durante o processo, além de desencadear potenciais problemas ambientais, exigindo investimentos significativos em tratamentos para controlar a poluição [2].

Os resíduos gerados no processamento da laranja para produção de suco consistem de laranjas descartadas, casca, semente, borra de extração de óleo essencial, polpa lavada, que podem ser denominados, genericamente de "bagaço". À medida que o bagaço da laranja representa cerca de $50 \%$ do total da fruta, o seu uso no desenvolvimento de produtos de alto valor agregado constitui uma alternativa interessante para o aproveitamento desse resíduo e solução de parte das preocupações econômicas, sociais e ambientais.

Atualmente, o principal uso desse resíduo está vinculado à fabricação de ração animal, devido ao seu alto valor nutricional, no entanto, outras aplicações incluem a extração de óleos essenciais, a fabricação de fertilizantes orgânicos e de fibras alimentares, o uso como adsorvente no tratamento de efluentes têxteis, bem como biomassa para a geração de energia [3].

As grandes quantidades de resíduos provenientes da indústria cítrica e o seu baixo custo favorecem o seu emprego como um potencial substrato biomássico energético. Entretanto, o elevado teor de umidade do bagaço de laranja (entre 75 e $82 \%$ b.u.) limita sua aplicação como combustível alternativo, devido aos altos custos de transporte, às dificuldades de armazenamento e à redução da eficiência térmica do material durante os processos conversão de energia [3]. Segundo Kaliyan e Morey [4], antes da compactação e conversão de energia a umidade de diferentes biomassas deve ser reduzida, mediante um processo de secagem, para valores abaixo de $10 \%$ b.u.

A secagem é, portanto, uma etapa essencial em qualquer uma das tecnologias de conversão térmica de biomassa, atuando como pré-tratamento para melhorar o desempenho dos processos de pirólise, gaseificação e combustão, além de preservar a biomassa em períodos relativamente longos de armazenamento.

A tecnologia de leito fluidizado vem sendo extensivamente aplicada para a secagem de diversos tipos de biomassa, tais como bagaço de azeitona [5], bagaço de beterraba [6] e bagaço de cana-de-açúcar [7]. As vantagens da técnica incluem a rápida mistura de sólidos, distribuição uniforme de temperatura, altas taxas de transferência de calor e massa devido a um melhor contato fluido-partícula e partícula-partícula, facilidade de automação, de operação e manutenção, bem como de ampliação de escala [8].

Entretanto, ainda não existem informações disponíveis na literatura sobre a secagem do bagaço de laranja em leito fluidizado, o que motiva o seu estudo. Para tanto, o conhecimento das características fluidodinâmicas das partículas é de fundamental importância para que os parâmetros do seu processamento, tais como: diâmetro e carga de partículas, e velocidade do ar, possam ser adequadamente definidos. Ademais, a fluidização de partículas de bagaço de laranja representa um desafio, devido à sua natureza heterogênea, ao seu tamanho, forma e densidade irregulares, bem como à sua elevada umidade.

Apesar de sua importância, poucos são os trabalhos realizados para investigar a fluidodinâmica de partículas de biomassa em leito fluidizado, tampouco os efeitos de suas propriedades físicas. Diante desse contexto, o objetivo deste trabalho foi estudar o comportamento fluidodinâmico de partículas de biomassa provenientes do bagaço de laranja em leito fluidizado convencional, avaliando como os parâmetros de fluidização foram influenciados pelo tamanho de partícula e pela carga de material alimentado no leito.

\section{MATERIAL E MÉTODOS}

\subsection{Material}

O bagaço da laranja utilizado neste trabalho foi fornecido por restaurantes situados nas imediações da Universidade Federal de Sergipe. A biomassa coletada foi triturada em um liquidificador industrial BM42-NR da marca BERMAR, e posteriormente classificada em um conjunto de peneiras (BETEL) auxiliado por agitador magnético. 
A umidade inicial do bagaço in natura foi determinada pelo método da estufa a $105^{\circ} \mathrm{C}$ até massa constante, cujo valor ficou em torno de $75 \%$ b.u. Para a preservação das características do material até o seu uso, cada fração granulométrica do bagaço foi acondicionada em sacos herméticos e armazenada em freezer doméstico a $5^{\circ} \mathrm{C}$.

Na Figura 1 pode ser observada a natureza heterogênea das partículas obtidas a partir do bagaço de laranja.

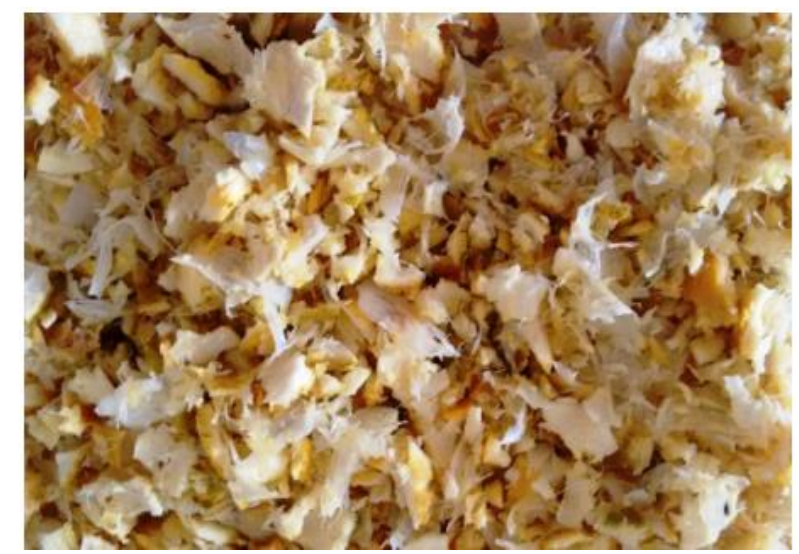

Figura 1: Bagaço de laranja triturado.

\subsection{Aparato experimental}

O estudo da fluidodinâmica do bagaço de laranja foi realizado numa unidade de leito fluidizado convencional, conforme esquematizado na Figura 2.

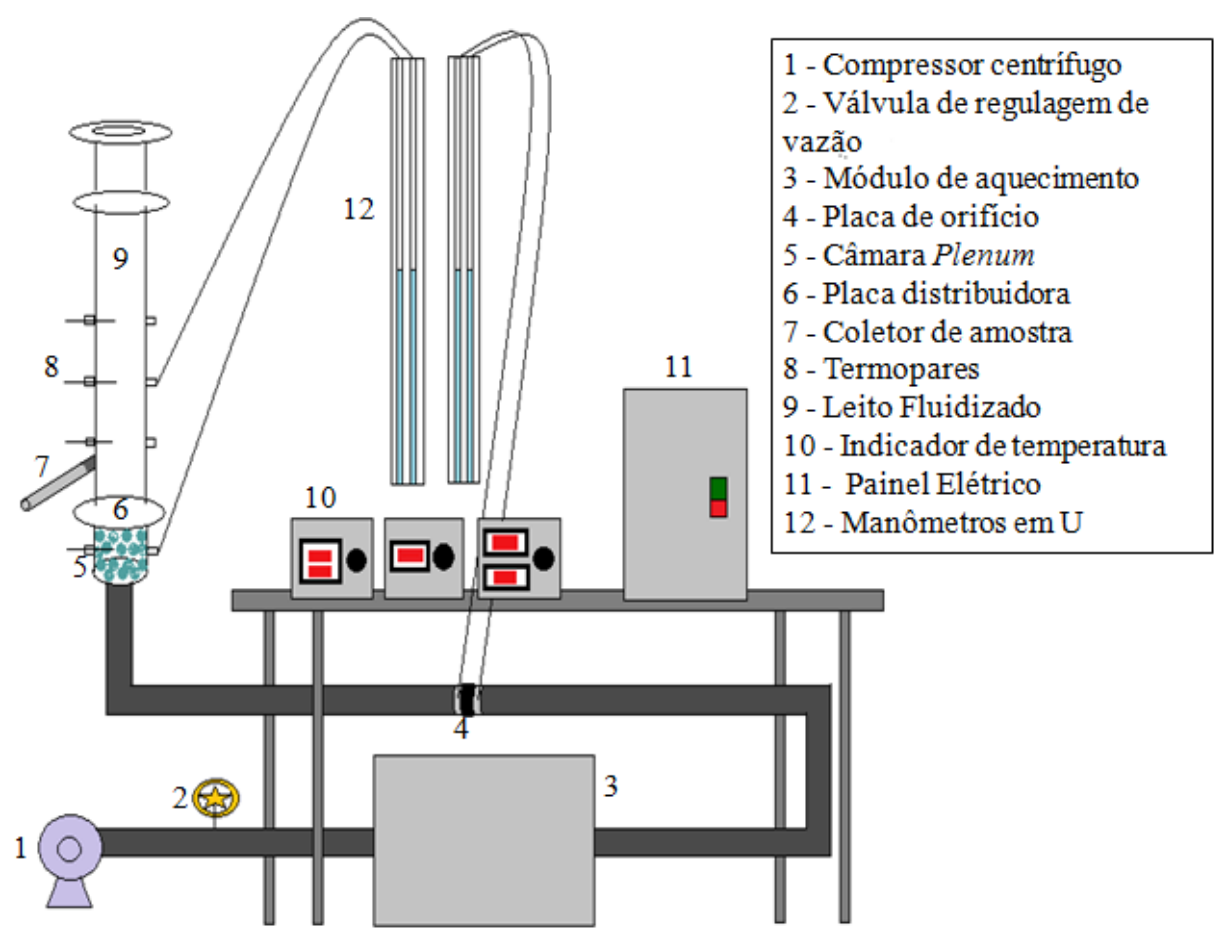

Figura 2: Aparato experimental.

Nesta unidade, o fluxo de ar é fornecido através de uma tubulação de 2 in por um compressor centrífugo (1) de $4 \mathrm{cv}$ (marca Eberle, modelo B 100, $3470 \mathrm{rpm}$ ) e regulado por meio de uma 
válvula gaveta (2). A medida da vazão do ar é realizada através da leitura da diferença de pressão em um manômetro em U (12) com água como fluido manométrico, acoplado a um medidor de vazão do tipo placa de orifício (4) previamente calibrado. $\mathrm{O}$ ar nos experimentos de secagem é aquecido, por meio de um aquecedor elétrico de $4500 \mathrm{~W}$ (3), passando por uma câmara plenum (5) e uma placa distribuidora (6), com percolação através do leito de partículas (9) e exaustão para a atmosfera. Cabe ressaltar que o módulo de aquecimento do ar permaneceu desligado durante os ensaios fluidodinâmicos.

A configuração do leito fluidizado estudada é a do tipo convencional, constituída de uma coluna cilíndrica de acrílico transparente para visualização do regime de fluidização, com diâmetro interno de $0,1085 \mathrm{~cm}$ e altura de $0,5 \mathrm{~m}$. A câmara plenum com $0,15 \mathrm{~m}$ de altura, recheada com esferas de vidro de mesmo tamanho, é acoplada ao leito por meio de flanges. No flange que separa o plenum do leito foi instalado um distribuidor do tipo placa perfurada, com arranjo triangular, para assegurar um escoamento uniforme do ar.

A queda de pressão total do sistema foi medida utilizando-se um manômetro diferencial, com tomadas de pressão localizadas $0,03 \mathrm{~m}$ abaixo e $0,48 \mathrm{~m}$ acima do distribuidor de ar.

Para medidas de temperatura foram utilizados quatro termopares do tipo PT-100, sendo um deles inserido na região da câmara plenum a $0,025 \mathrm{~m}$ da placa distribuidora para medir a temperatura do ar de entrada, e os outros três dispostos ao longo do leito.

\subsection{Caracterização física do material}

\subsubsection{Distribuição granulométrica}

A distribuição do tamanho de partículas foi determinada pela técnica de peneiramento, utilizando as peneiras Tyler de mesh 3,5, 4, 5, 6, 7, 8, 9, 10, 12 e 14, e uma massa de material em torno de $4 \mathrm{~kg}$. Após 20 minutos de agitação, foram pesadas as massas de partículas retidas em cada peneira do conjunto, a fim de calcular a fração mássica retida e obter a distribuição de frequência do tamanho de partículas.

\subsubsection{Densidade real das partículas $\left(\rho_{p}\right)$}

A densidade real do material foi determinada utilizando um picnômetro a gás hélio, modelo AccuPyc II 1340, da marca Micromeritics.

\subsubsection{Densidade de empacotamento do leito $\left(\rho_{b}\right)$}

A densidade de empacotamento do leito foi determinada a partir da razão entre a massa de partículas alimentadas e o volume ocupado por elas no leito.

\subsubsection{Porosidade $(\varepsilon)$}

A porosidade do leito foi calculada a partir da relação entre a densidade de empacotamento do leito e a densidade real da partícula:

$$
\varepsilon=1-\frac{\rho_{b}}{\rho_{r}}
$$

\subsection{5 Ângulo de repouso}

O ângulo de repouso foi determinado pelo modo estático, no qual, $0,4 \mathrm{~kg}$ de bagaço de laranja foram descarregados através de um funil com as seguintes dimensões: $0,02 \mathrm{~m}$ de diâmetro menor, $0,245 \mathrm{~m}$ de diâmetro maior e altura de $0,315 \mathrm{~m}$. O funil foi posicionado a uma altura de $0,15 \mathrm{~m}$ da superfície plana, e a partir da queda livre das partículas, foi formado um amontoado suficiente para determinar experimentalmente o ângulo de repouso estático em relação ao plano horizontal. 


\subsection{Análise fluidodinâmica do leito}

Os ensaios fluidodinâmicos foram conduzidos com cargas de leito de 400, 700 e $1000 \mathrm{~g}$, e partículas de bagaço de laranja com diâmetros médios de 1,55, 1,85 e 2,58 mm. A fluidização das partículas foi obtida utilizando ar a uma temperatura média de $28^{\circ} \mathrm{C}$.

Com o intuito de minimizar os efeitos de empacotamento das partículas sobre as curvas características dos leitos formados, procedeu-se uma movimentação inicial das mesmas através do aumento da velocidade do ar, até a condição de fluidização incipiente, quando a vazão de ar foi bruscamente interrompida, desencadeando num empacotamento frouxo das partículas.

Após o empacotamento das partículas, a altura inicial do leito estático foi medida numa escala graduada fixada na coluna. Em seguida, o soprador foi ligado e a válvula de regulagem de vazão aberta lenta e gradualmente, registrando-se por meio dos manômetros em $U$ os dados de queda de pressão na placa de orifício e no sistema leito-distribuidor até que se observasse uma fluidização plena. Também foram medidas em cada vazão do ar a altura do leito e as temperaturas na entrada e no topo do leito. As mesmas medidas foram realizadas em vazões decrescentes do ar, no processo de defluidização.

Tomou-se o cuidado para que os ensaios durassem o menor tempo possível, a fim de evitar uma perda de umidade significativa do material. Para quantificar tal perda, foi determinado o teor de umidade do material antes e depois do experimento, pelo método da estufa a $(105 \pm 3)^{\circ} \mathrm{C}$.

A partir do levantamento das curvas características de queda de pressão do leito em função da velocidade superficial do ar foram determinados graficamente os parâmetros de queda de pressão máxima, queda de pressão de mínima fluidização e velocidade de mínima fluidização $\left(\mathrm{v}_{\mathrm{mf}}\right)$. A qualidade da fluidização foi também avaliada com base nos cálculos do número de Froude (Fr) e do índice de efetividade de fluidização (IF), expressos pelas Equações (2) e (3), respectivamente.

$$
\begin{gathered}
F r=\frac{v_{m f}^{2}}{g d_{p}} \\
I F=\frac{-\Delta P}{m_{b} g / A}
\end{gathered}
$$

sendo $g$ a aceleração da gravidade, $d_{p}$ o diâmetro de peneira da partícula, A a área da seção transversal do leito, $v_{m f}$ a velocidade mínima de fluidização; $-\Delta \mathrm{P}$ a queda de pressão registrada no leito e $\mathrm{m}_{\mathrm{b}}$ a massa de material particulado no leito.

Para $F r>1$, ocorre fluidização do tipo agregativa e $F r<1$ indica a ocorrência da fluidização particulada. Por sua vez, o índice de efetividade da fluidização $(I F)$ permite mensurar o percentual do material particulado efetivamente fluidizado.

\section{RESULTADOS E DISCUSSÃO}

\subsection{Caracterização física do material}

\subsubsection{Distribuição granulométrica}

O resultado típico da distribuição de tamanho das partículas via peneiramento é apresentado na Figura 3. Verifica-se que a faixa predominante de tamanho de partículas está situada entre 2,36 e $2,8 \mathrm{~mm}$, para a qual foram determinadas a densidade real das partículas, a densidade de empacotamento e a porosidade do leito. 


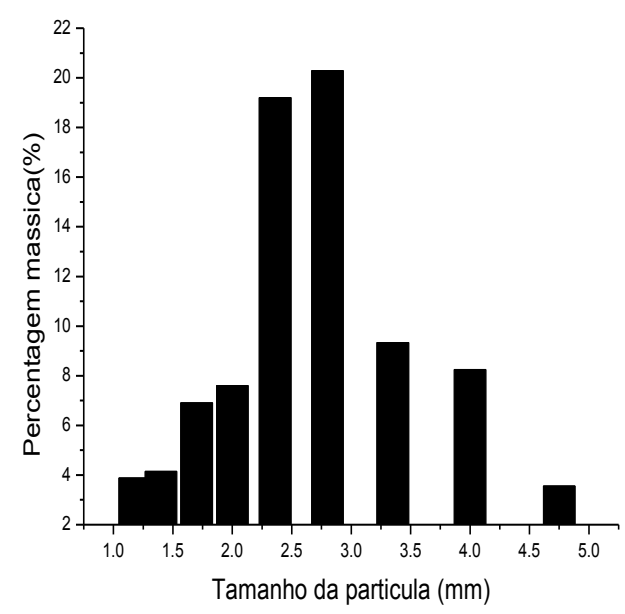

Figura 3: Curva de distribuição granulométrica para o bagaço de laranja triturado.

\subsubsection{Propriedades físicas das partículas e do leito}

A densidade real das partículas, determinada via picnometria a gás, foi de $1,1776 \mathrm{~g} / \mathrm{cm}^{3}$. Os resultados de densidade e a porosidade do leito para cada carga de material empregada estão listados na Tabela 1.

Tabela 1: Densidade e porosidade do leito para diferentes cargas de material.

\begin{tabular}{ccccc}
\hline $\mathbf{m}(\mathbf{g})$ & $\mathbf{H}(\mathbf{c m})$ & $\mathbf{V}\left(\mathbf{c m}^{\mathbf{3}}\right)$ & $\boldsymbol{\rho}_{\text {leito }}\left(\mathbf{g} / \mathbf{c m}^{\mathbf{3}}\right)$ & $\boldsymbol{\varepsilon}(-)$ \\
\hline 400 & 10 & 924,59 & 0,433 & 0,596 \\
700 & 16 & 1479,34 & 0,473 & 0,558 \\
1000 & 22 & 2034,10 & 0,492 & 0,541 \\
\hline
\end{tabular}

Nota-se que os valores de porosidade dos leitos estáticos, em todas as cargas utilizadas, foram significativamente maiores que 0,40 , valor comumente reportado para leitos fixos. Isto pode ser atribuído à técnica de empacotamento do tipo frouxo empregada nos experimentos. Além disso, verifica-se uma tendência dos leitos rasos apresentarem uma menor densidade de empacotamento e se caracterizarem por uma maior porosidade, o que está de acordo com a literatura.

\subsection{4 Ângulo de repouso estático}

O ângulo de repouso estático das partículas de bagaço com diâmetro médio de 2,58 $\mathrm{mm}$ teve o valor de $37,34^{\circ}$. Esse valor encontra-se na faixa de ângulo que classifica as partículas como um material de aceitável escoabilidade, segundo Aulton [9].

\subsubsection{Classificação de Geldart}

A partir dos dados de massa específica e diâmetro da partícula o material de estudo pôde ser classificado como uma partícula do tipo D, segundo Geldart [8]. Partículas deste grupo são grandes e/ou com alta densidade, caracterizadas por regimes de fluidização com sluggings e/ou canais preferenciais, e baixas expansões do leito.

\subsection{Análise fluidodinâmica}

Na Figura 4 são apresentados os resultados típicos de queda de pressão no leito $(\Delta \mathrm{P})$ em função das vazões crescentes e decrescentes do ar ( $\left.Q_{\text {ar }}\right)$ obtidos para o bagaço de laranja com partículas de diâmetro médio de $2,58 \mathrm{~mm}$, nos ensaios realizados com a carga de $400 \mathrm{~g}$. Pode-se observar um comportamento típico de leito fluidizado, com a presença de uma região de leito fixo, com a queda de pressão aumentando linearmente com a velocidade do ar, e uma região de leito fluidizado, na qual a queda de pressão no leito permanece praticamente constante com o aumento 
da velocidade do ar. Na defluidização os valores de queda de pressão na região de leito fixo são menores que aqueles medidos em vazões crescentes, o que pode ser explicado pela acomodação das partículas na forma de um leito expandido, com porosidade maior do que aquela do leito fixo inicial.

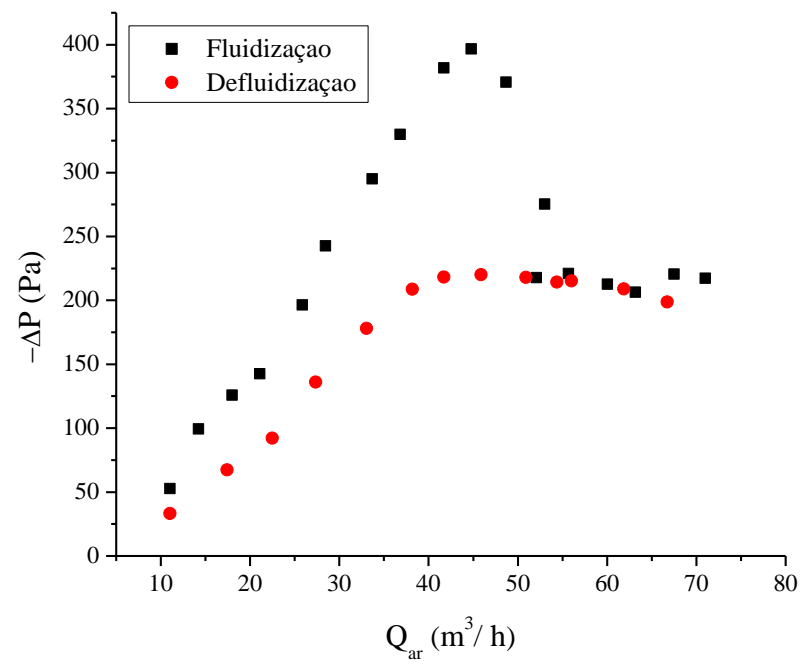

Figura 4: Queda de pressão em função da vazão do ar para partículas de diâmetros médios de 2,58 mm, carga de $400 \mathrm{~g}, X=75 \%$ b.u.

Nota-se ainda uma redução brusca da queda de pressão na região de transição entre o leito fixo e o leito fluidizado, que pode estar associada à canalização do ar dentro do leito devido à alta coesividade das partículas, causada pela elevada umidade superficial das mesmas. O mesmo comportamento foi observado por Duan et al. [10], ao trabalharem com uma mistura de nanopartículas de $\mathrm{SiO}_{2}$ e $\mathrm{TiO}_{2}$, que devido às forças interpartícula e eletrostática, resultou em uma redução brusca da queda de pressão no leito, antes da fluidização plena.

Deve ser ressaltado que após as forças coesivas intrapartículas terem sido superadas e o leito ter se expandido, com o aumento da vazão de ar os canais foram eliminados e um bom comportamento de fluidização foi observado visualmente. Este bom comportamento é corroborado pelas baixas flutuações da queda de pressão no leito para velocidades decrescentes do ar, o que é indicativo de uma fluidização sem a formação de "slugs", grandes bolhas de ar formadas pela coalescência de bolhas menores, cujo diâmetro é equivalente ao diâmetro do leito e que se movimentam num fluxo pistonado.

Os teores de umidade das amostras foram determinados antes e depois dos testes fluidodinâmicos, sendo possível observar que estes variaram de $75 \%$ b.u. para $72 \%$ b.u. para a amostra de bagaço. Variação que está associada à evaporação da água, mas sem influência significativa sobre as curvas características obtidas.

A Figura 5 mostra as curvas características das partículas do bagaço com diferentes diâmetros médios (dp), para uma carga de $400 \mathrm{~g}$ e umidade inicial de $75 \%$ b.u.

Nota-se que com o aumento do tamanho de partícula, há uma menor inclinação da reta correspondente à região de leito fixo, devido ao aumento da permeabilidade do leito, que faz com que a resistência à passagem do ar seja menor, e consequentemente a queda de pressão no leito diminua. Já na fluidização plena há uma convergência para o mesmo patamar, visto que a mesma carga de material foi alimentada no leito, de modo que o seu peso aparente permanece o mesmo, independentemente do tamanho de partícula.

Alguns parâmetros de grande importância para análise da fluidodinâmica do leito, tais como, a velocidade de mínima fluidização, a queda de pressão de mínima fluidização, a queda de pressão máxima, o número de Froude e o Índice de fluidização foram determinados e encontram-se apresentados na Tabela 2. 


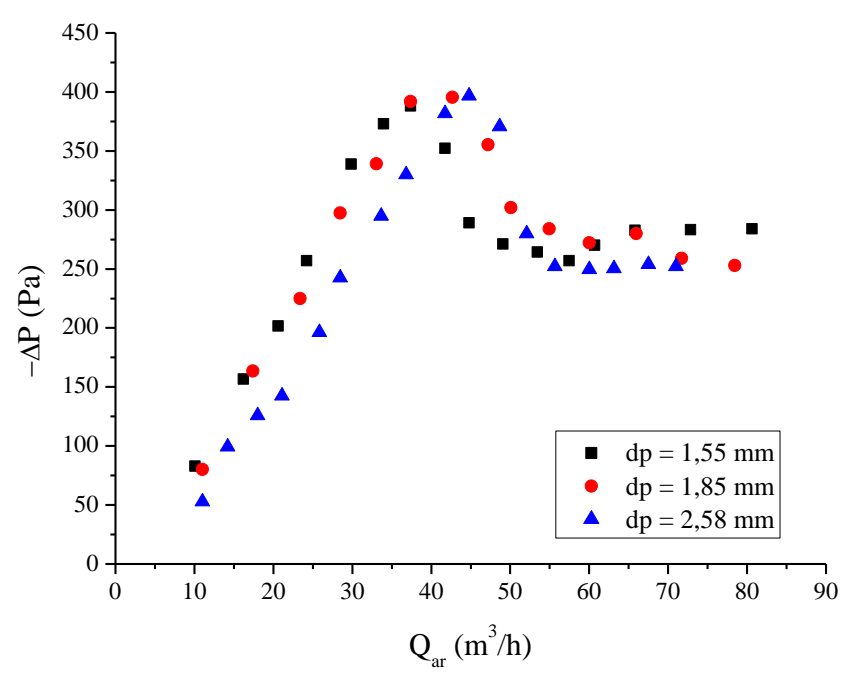

Figura 5: Queda de pressão em função da vazão do ar para diferentes diâmetros médios de partículas, carga de 400 g e umidade de $75 \%$ b.u.

Tabela 2: Parâmetros fluidodinâmicos dos leitos constituídos por diferentes tamanhos de partículas de biomassa provenientes das amostras de bagaço de laranja, carga de $400 \mathrm{~g} e$ umidade de $75 \%$ b.u.

\begin{tabular}{cccccc}
\hline $\begin{array}{c}\text { Diâmetro médio } \\
(\mathbf{m m})\end{array}$ & $\boldsymbol{v}_{\mathbf{m f}}(\mathbf{m} / \mathbf{s})$ & $-\Delta \mathbf{P}_{\mathbf{m f}}(\mathbf{P a})$ & $\boldsymbol{- \Delta} \mathbf{P}_{\max }(\mathbf{P a})$ & $\mathbf{F r}$ & $\mathbf{I F}(\boldsymbol{\%})$ \\
\hline 2,58 & 1,83 & 292 & 400 & 114,19 & 68,8 \\
1,85 & 1,53 & 268 & 395 & 128,99 & 63,1 \\
1,55 & 1,36 & 276 & 384 & 121,64 & 65,0 \\
\hline
\end{tabular}

A partir dos resultados listados na Tabela 2 verifica-se que a velocidade de mínima fluidização aumentou de $1,36 \mathrm{~m} / \mathrm{s}$ para $1,83 \mathrm{~m} / \mathrm{s}$, com o aumento do diâmetro médio da partícula de 1,55 para $2,58 \mathrm{~mm}$. Esse aumento em torno de $35 \%$ em $v_{\mathrm{mf}}$ está coerente com a teoria, visto que partículas de maior diâmetro precisam de uma maior energia cinética para provocar o seu deslocamento, e consequentemente, tirar o leito de sua inércia. Comportamentos similares foram observados por Borel [11] na secagem em leito fluidizado do pólen apícola variando-se o diâmetro de 0,84 $\mathrm{mm}$ a $2,86 \mathrm{~mm}$.

Por sua vez, os desvios dentro dos valores obtidos para as quedas de pressão máxima e mínima foram inferiores aos erros de medida, de modo que não houve uma influência significativa do diâmetro de partículas sobre tais parâmetros.

O número de Froude para as partículas com diferentes diâmetros ficou na faixa de 114,2 a 121,6, indicando que a fluidização das partículas do bagaço de laranja foi do tipo agregativa, caracterizada por um fenômeno semelhante à ebulição, com formação de bolhas de gás que atravessam o leito sólido e rompem-se na superfície, empurrando as partículas de sólido para cima. Também pode ser verificado que as partículas de maior diâmetro, 2,58 mm, apresentaram o maior valor do índice de fluidização $(68,8 \%)$ e menor valor para o número de Froude $(114,2)$, sugerindo uma menor dificuldade para a fluidização, o que foi constatado visualmente.

Segundo Marring et al. [12], o contato gás-sólido e a condição em que se encontram as partículas suportadas pelo fluxo de gás são medidas de "qualidade" da fluidização. Se a queda de pressão do leito for aproximadamente igual ao peso do leito por unidade de área da seção transversal do leito tem-se então uma fluidização de boa qualidade, porém quando as forças interpartículas no leito causam formação de canais preferenciais do gás, a queda de pressão poderá ser muito baixa, indicando uma fluidização pobre. Delebarre et al. [13] explica que um valor do índice de efetividade da fluidização (IF) próximo da unidade corresponde à situação em que o peso total do leito está fluidizado, sem zonas mortas ou efeito de canalização. Porém, deve ser ressaltado que os elevados valores de IF reportados na literatura se referem a partículas secas. 
Assim, os valores de IF obtidos para o bagaço de laranja, em torno de $65 \%$, podem ser atribuídos à natureza coesiva e à alta umidade do material trabalhado.

Os resultados de queda de pressão em função da velocidade superficial do ar para diferentes massas iniciais de partículas alimentadas no leito estão apresentados na Figura 6. Na Tabela 3 são listados os valores obtidos para os correspondentes parâmetros de fluidização.

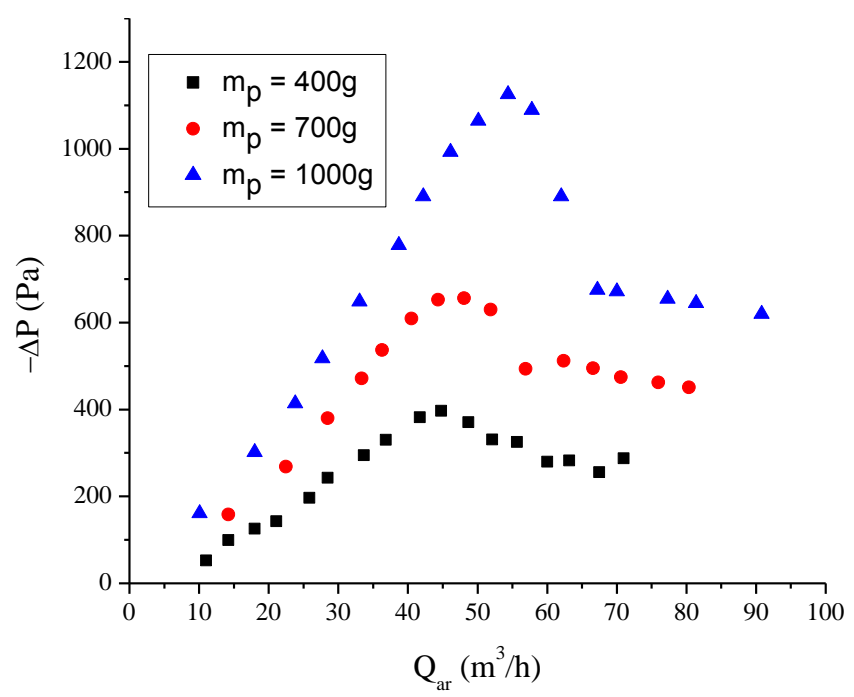

Figura 6: Queda de pressão em função da vazão do ar para diferentes cargas de partículas com diâmetro médio de $2,58 \mathrm{~mm}$ e umidade de $75 \%$ b.u.

Tabela 3: Parâmetros fluidodinâmicos obtidos para diferentes cargas de partículas com diâmetro médio de $2,58 \mathrm{~mm}$ e umidade de $75 \%$ b.u.

\begin{tabular}{ccccc}
\hline Massa $(\mathbf{g})$ & $\boldsymbol{v}_{\mathbf{m f}}(\mathbf{m} / \mathbf{s})$ & $\boldsymbol{- \Delta} \mathbf{P}_{\mathbf{m f}}(\mathbf{P a})$ & $\boldsymbol{- \Delta} \mathbf{P}_{\max }(\mathbf{P a})$ & $\mathbf{I F}(\boldsymbol{\%})$ \\
\hline 400 & 1,83 & 292 & 400 & 68,8 \\
700 & 1,9 & 474 & 661 & 63,82 \\
1000 & 2,04 & 661 & 1123 & 62,3 \\
\hline
\end{tabular}

Com base na Figura 6 nota-se um aumento significativo da queda de pressão no leito com o aumento da carga de partículas, tanto na região de leito fixo, como na região de leito fluidizado. Tal resultado pode ser explicado pelo aumento da altura do meio poroso e do consequente aumento das forças resistivas fluido-partícula dentro do leito.

$\mathrm{O}$ aumento da carga de partículas de 400 para $1000 \mathrm{~g}$ resultou em aumentos de aproximadamente 180 e $126 \%$ nos valores de queda de pressão máxima e de mínima fluidização. Ao analisar as diferenças entre os valores desses parâmetros verifica-se que quanto maior a carga alimentada no leito, maior a diferença entre $\Delta \mathrm{P}_{\max }$ e $\Delta \mathrm{P}_{\mathrm{mf}}$, o que evidencia uma redução mais brusca de queda de pressão no período de transição de leito fixo para leito fluidizado, e, consequentemente, um maior efeito da formação de canais preferenciais dentro do leito. Isto explica o menor índice de efetividade de fluidização $(62,3 \%)$ para a maior carga, de $1 \mathrm{~kg}$

A partir dos dados apresentados na Tabela 3, verifica-se que o aumento da quantidade de material no leito não influenciou de forma significativa a velocidade de mínima fluidização, visto que o valor médio de $\mathrm{v}_{\mathrm{mf}}$ foi de $1,92 \mathrm{~m} / \mathrm{s}$, com desvio de apenas $0,11 \mathrm{~m} / \mathrm{s}$.

\section{CONCLUSÃO}

O tamanho de partícula predominante nas amostras cominuídas de bagaço de laranja foi o de $2,58 \mathrm{~mm}$. A densidade real foi de $1,1776 \mathrm{~g} / \mathrm{cm}^{3}$. De acordo com a classificação de Geldart, o material foi identificado como partículas do tipo $\mathrm{D}$, cujas principais características são a formação de canais preferenciais e a baixa expansão do leito.

A porosidade dos leitos estáticos apresentou valores elevados para todas as cargas utilizadas 
devido à técnica de empacotamento do tipo frouxo empregada. Além disso, verificou-se uma tendência dos leitos rasos apresentarem uma menor densidade de empacotamento e uma maior porosidade.

Partículas do bagaço de laranja apresentaram valores de ângulo de repouso estático em torno de $37^{\circ}$, caracterizando-se como um material de aceitável escoabilidade.

O bagaço de laranja mesmo tendo um caráter coesivo, conseguiu visivelmente fluidizar em leito fluidizado convencional, conforme evidenciado pelas curvas fluidodinâmicas características.

A velocidade de mínima fluidização $\left(\mathrm{v}_{\mathrm{mf}}\right.$ ) aumentou de 1,36 para $1,83 \mathrm{~m} / \mathrm{s}$ ao se aumentar o diâmetro médio das partículas de bagaço de laranja de 1,55 para 2,58 $\mathrm{mm}$.

Apesar dos leitos com partículas de maior diâmetro demandarem uma maior velocidade do ar para fluidizar, o diâmetro médio das partículas de bagaço de laranja recomendado para a fluidização é o de $2,58 \mathrm{~mm}$, tendo em vista o maior índice de fluidização e o menor número de Froude obtidos.

A queda de pressão na condição de mínima fluidização aumentou em torno de $126 \%$ com o aumento de $0,4 \mathrm{~kg}$ para $1 \mathrm{~kg}$ na carga de partículas de 2,58 $\mathrm{mm}$ alimentadas no leito. Entretanto, não houve influência significativa da carga de partículas sobre a velocidade de mínima fluidização.

Para a secagem em leito fluidizado das partículas de $2,58 \mathrm{~mm}$ do bagaço de laranja, independentemente da carga a ser processada, a velocidade do ar requerida para a operação deve ser igual ou superior a $(1,92 \pm 0,11) \mathrm{m} / \mathrm{s}$.

\section{AGRADECIMENTOS}

Os autores agradecem ao CNPq e CAPES pelo auxílio financeiro, bem como ao LQI/UFS pela realização da etapa de cominuição do bagaço de laranja.

\section{REFERÊNCIAS BIBLIOGRÁFICAS}

1. Citrusbr,disponívelem:<http://www.citrusbr.com.br/download/biblioteca/o_retrato_da_citricultura_brasi leira_ba xa.pdf $>$, data de acesso 03/03/2015.

2. Pelizer LH, Pontieri MH, Moraes IO. Utilização de resíduos agroindustriais em processos biotecnológios como perspectiva de redução do impacto ambiental. J. of Technol. Manage.Innov. 2007 Mar; 2(1):118-127, disponível em <http://www.jotmi.org/index.php/GT/article/view/art41>.

3. Liu Y, Peng J, KanshaY, Ishizuka M, Tsutsumi A, Jia D, Bi XT, Lim CJ, Sokhansanj S. Novel fluidized bed dryer for biomass drying. Fuel Process. Technol. 2014 Jun; 122:170-175, doi:10.1016/j.fuproc.2014.01.036.

4. Kaliyan N, Morey RV. Factors affecting strength and durability of densified biomass products. Biomass and Bioenergy 2009 Mar; 33(3): 337-359, doi: 10.1016/j.biombioe.2008.08.005.

5. Meziane S. Drying kinetics of olive pomace in a fluidized bed dryer. Energy Convers. Manage. 2011 Mar; 52(3):1644-1649, doi:10.1016/j.enconman.2010.10.027.

6. Polanco LS, Kochergin V, Alvarez JF. Fluidized Bed Superheated Steam Dryer for Bagasse: Effects of Particle Size Distribution. J. Sustain. Bioenergy Syst. 2013 Dec; 3(4):265-271, doi: 10.4236/jsbs.2013.34036.

7. Meirelles AJA. Secagem do bagaço de cana em leito fluidizado [Dissertação de Mestrado]. UNICAMP, Campinas (SP): Universidade Estadual de Campinas, 1984.

8. Geldart D. Gas Fluidization Technology. New York: Jonh Wiley \& Sons Ltd.; 1986. 468p.

9. Aulton ME. Delineamento de Formas Farmacêuticas. Porto Alegre: Artmed, 2005.

10. Duan H, Wang J, Zhou T. Fluidization of mixed $\mathrm{SiO} 2$ and $\mathrm{TiO} 2$ nanoparticles with FCC coarse particles. Procedia Eng. 2015 Apr;102:815-820, doi:10.1016/j.proeng.2015.01.196.

11. Borel LDMS. Aplicação de um secador de leito fluidizado assistido por aquecimento infravermelho para o beneficiamento de pólen apícola [Dissertação de Mestrado]. São Cristóvão (SE): Universidade Federal de Sergipe, 2014. 139 p.

12. Marring E, Hoffmann AC, Janssen LPBM. The effect of vibration on the fluidization behavior of some cohesive powders. Powder Technol. 1994 Apr; 79(1):1-10, doi:10.1016/0032-5910(94)02810-9.

13. Delebarre A, Morales J, Ramos L. Influence of the bed mass on its fluidization characteristics. Chem. Eng. J. 2004 Mar; 98(1-2): 81-88, doi:10.1016/S1385-8947(03)00206-7. 